\title{
Artikel
}

Patrick Stoffel*

\section{Deep time Heimat. Die prähistorischen Landschaften des Deutschen Reichs}


Abstract: Im Deutschen Reich avancierte die Landschaft zum zentralen Medium des Heimaterlebens. Dabei erfuhr die Heimat mittels der Erfahrung von fremden und exotischen Landschaften in den Kolonien eine nie dagewesene räumliche Expansion: aus der Ferne konnte die Nation selbst zur Heimat werden. Während diese räumliche, Dehnbarkeit' der Heimat (und deren identitätsstiftende Funktion) schon seit Längerem Gegenstand der Forschung ist, erfuhr die zeitliche Dimension der Heimat bislang wenig Aufmerksamkeit. Obwohl die Entdeckung und Entfaltung der geologischen Zeitskala im 19. Jahrhundert neue, unvorstellbar große Zeiträume bereitstellte, blieben der Heimat scheinbar enge zeitliche Grenzen gesetzt. Dieser Beitrag zeigt anlässlich der Untersuchung populärwissenschaftlicher Schriften aus den Jahren 1898-1931 von Alfred Götze und Wilhelm Bölsche, dass im Deutschen Reich parallel zur räumlichen Expansion der Heimat auch eine zeitliche Expansion erfolgte, die über die Verortung der Heimat in der , guten alten Zeit' weit hinausreichte und über die Ur- und Frühgeschichte bis tief in die Erdgeschichte ausgriff. Im Medium prähistorischer Landschaften erschloss sich den Zeitgenossen eine in zwei Versionen vorliegende deep time Heimat. Eine nationalistische deep time Heimat verortete die nationale Einheit in der Tiefe der Zeit und beförderte damit ein umfassenderes und zugleich abstrakteres, ein ,nationales' Heimaterleben. Eine imperialistische deep time Heimat diente als Anschauungsfall für den ,Kampf ums Dasein' und wies die Expansion der Heimat als einzig möglichen Weg zu ihrer Bewahrung aus.

In the German Reich, the landscape became the central medium to experience Heimat. The exotic landscapes the colonies provided led to an unprecedented spatial expansion: from a distance, the nation itself could become Heimat. While this spatial 'extensibility' as well as its capability to establish identity has long been the subject of research, so far the temporal dimension of Heimat has received little attention. The new and unimaginably long periods of time the discovery and exposition of the geological time scale in the 19th century provided seemed not to affect the Heimat. Examining popular scientific writings from the years 1898-1931 by Alfred Götze and Wilhelm Bölsche, this article shows that in the German Reich along with the spatial expansion of Heimat a temporal expansion took place. Including prehistory and earth history, this temporal expansion reached far beyond locating the Heimat in the 'good old days'. In the medium of prehistoric landscapes, the contemporaries could experience two versions of a deep time Heimat. A nationalist deep time Heimat localized national unity in the depths of time, thus promoting a more comprehensive and at the same time more abstract 'national' Heimat. An imperialist deep time Heimat helped to expose the 'struggle for existence' and promoted the expansion of the Heimat as the only possible way to preserve it.

Keywords: Urzeit, Deep-Time, Popular Science, Heimat und Erdgeschichte, Imperialismus, Nationalismus

*Dr. Patrick Stoffel, Leuphana Universität Lüneburg, Institut für Geschichtswissenschaft und Literarische Kulturen, Professur für Kulturgeschichte des Wissens, E-Mail: Patrick.Stoffel@leuphana.de

\section{Willy Hellpach. Vom Heimweh nach exotischen Landschaften}

"Heimat", schrieb Willy Hellpach, „erlebt der schlichte Mensch erst in der Fremde" (1935, 219). Dieser eine, prägnante Satz reichte dem Mediziner, Psychologen und Politiker Willy Hellpach (1877-1955), der über viele Jahrzehnte hinweg den Einfluss von Wetter, Klima und Landschaft, später auch des Bodens, auf das Seelenleben des Menschen erforschte, den Vorgang des Heimater- lebens der populären „Blut-und-Boden"-Ideologie zu entziehen. Dem zeitgenössischen Diskurs, der die Grenzen zwischen geistiger und biologischer Milieuabhängigkeit gerade auch mittels des Heimatbegriffs immer weiter auflöste (vgl. Güttler 2017), wollte und konnte er sich jedoch nicht verweigern. Der vierten, unter dem Titel Geopsyche erschienenen Auflage der erstmals 1911 unter dem Titel Die geopsychischen Erscheinungen veröffentlichten Ergebnisse seiner Arbeit fügte er ein neues, mit dem Einfluss des Bodens beschäftigtes 
Kapitel hinzu, in welchem er die "Aufhellung der Bodenwesenhaftigkeit des Menschen" als "eine der großartigsten wissenschaftlichen Aufgaben des 20. Jahrhunderts" auswies. Er sah aber für die "Bodenhörigkeit" der Lebewesen als zentralen Lehrsatz der „Blut-und-Boden"-Ideologie, wie er vom Anthropologen und Rassentheoretiker Egon Freiherr von Eickstedt in den Satz "Keine Hominidenform verläßt ungestraft ihre angestammte Umwelt" gepresst wurde, keinerlei wissenschaftliche Belege (Hellpach 1935, 197 f.). Im Gegenteil wird ,Heimat', wie Hellpach schreibt, oft erst in der Fremde erlebt, und zwar gerade aufgrund ihres Verlustes. ,Heimat' steht folglich symptomatisch für das Aufbrechen der - ohne Gang in die Fremde - unhinterfragten Einheit von Person und Umwelt. Heimat, muss man mit Willy Hellpach feststellen, ist prekär.

Hellpach ist heute weitgehend vergessen. Der mit der Geschichte der Umweltpsychologie vertraute Rudolf Miller sieht den zentralen Grund hierfür darin, dass er in seinem Denken dem ausgehenden 19. Jahrhundert verhaftet blieb, was zu "kategorialen Unschärfen und nicht operationalisierbaren Konstrukten" führte $(2018,338)$. Gerade hierin aber liegt der Wert seiner Arbeit für einen kulturgeschichtlichen Zugang zum Heimaterleben im Deutschen Reich, diesem diffusen Vorgang, der sich um 1900 ebenso wenig wie heute durch begriffliche Schärfe auszeichnet.

Wenn Hellpachs Heimatbegriff darin vom zeitgenössischen Diskurs abwich, dass er sich von deterministischen Milieutheorien distanzierte, wie sie infolge von Friedrich Ratzels Anthropogeographie von zahlreichen Zeitgenossen vertreten wurden, so stimmte er mit ihm in dem Aspekt überein, dass er in der Landschaft das zentrale Medium des Heimaterlebens erkannte: "[S]ehr oft steht die heimatliche Landschaft durchaus im Vordergrunde dieses Erlebnisses." (Hellpach 1935, 219) Landschaft zählt Hellpach neben Wetter, Klima und Boden zu den wesentlichen Eigenschaften der natürlichen Umwelt. Diese bildet zusammen mit Gemeinschaft, Kultur und Technik die drei Umwelten, in die der Mensch eingelassen ist. Das Heimaterleben resultiert bei Hellpach folglich nicht primär aus dem Verlust (bzw. dem Wechsel) der sozialen oder der kulturell-technischen, als vielmehr aus dem Verlust der natürlichen Umwelt. Und von deren Eigenschaften ist es wiederum die (veränderte) Landschaft, die Hei- mat hervorbringt. Eine Landschaft aber vermag der Mensch erst dort zu erblicken, so Hellpach, wo ihm die Natur nicht länger Ressource ist, "ohne jeden Nutzzweck" (1935, 201). Ein derart als Landschaft erblicktes Stück Erde macht einen "Eindruck" auf den Menschen, ein Vorgang, den Hellpach vom "Einfluss" nehmen, wie er Grundlage des „Bodenhörigkeit"-Denkens ist, geschieden wissen willl $(1935,202)$. Das Landschaftserleben bedarf daher eines synthetischen Sehens, womit die Landschaft unter den Eigenschaften der natürlichen Umwelt eine Sonderstellung einnimmt.

Mit seinem geopsychischen Zugang zum Heimaterleben kommt Hellpach zum gleichen Ergebnis wie viele seiner Zeitgenossen auf anderen Wegen auch. Als organisches Fundament und wesentlicher Ausdruck heimatlicher bzw. nationaler Identität (wobei Heimat und Nation sowohl deckungsgleich als auch verschieden sein konnten) fungierte im Deutschen Reich, wie Thomas M. Lekan mit Imagining the Nation in Nature (2004) dargelegt hat, die Landschaft.

Aber nicht alle Landschaften nehmen, so Hellpach, den gleichen Einfluss: „Unter den Arten von Fremde ist es die exotische Landschaft, die das einfache Gemüt am elementarsten packt und seinem Heimweh eine oft siegreiche Widerkraft entgegenstellt." (1935, 219) Speziell die exotische Landschaft dient also nicht allein als Kontrastfolie, vor der die gewohnte, aber verlorene Landschaft als Heimat kenntlich wird, sondern sie kann das hieraus resultierende Heimweh überwinden und selbst zur ersehnten Heimat werden. Hellpach fährt fort:

Es gibt eine Art Heimweh nach exotischen Landschaf-
ten, sei es, daß sie im Fluge vorüberzogen, sei es, daß
man sich in ihnen wie in einer neuen Heimat einge-
richtet hatte; viele Nordländer sind das Heimweh nach
Sonnigkeit und Farbigkeit der Mittelmeernatur nie
wieder losgeworden. (1935, 219)

Habe das Heimweh nach einem ,Platz an der Sonne' zu Goethes Zeiten noch auf einer Reise in den "mittelmeerische[n] Süden" gestillt werden können, führe die Reise den Heimwehgeplagten heute in die Tropen. Damit übernimmt die ,fremde, exotische Landschaft' in Hellpachs Studie exakt jene doppelte Funktion, die der Historiker Jens Jäger (2009) den deutschen Kolonien um 1900 innewohnen sah: als Kontrastfolie, vor 
der die gewohnte, aber verlorene Landschaft als Heimat kenntlich wird, und als mögliche zukünftige Heimat.

Das prekäre Heimaterleben ist bei Hellpach zuvorderst Resultat räumlicher Mobilität, mit der die als natürlich gedachte Einheit von Person und Umwelt aufgelöst und damit infrage gestellt, aber eben auch verhandelbar wird. Mit den kolonialen Territorien des Deutschen Reichs, dessen kolonialen Bestrebungen mit dem Verlust der Kolonien infolge des Ersten Weltkriegs mitnichten endeten, erfuhr das Heimaterleben eine immense räumliche Ausdehnung. Hierfür bedurfte es gar nicht der massenhaften Ausreise von Deutschen in die Kolonien - was tatsächlich nicht der Fall war -, es reichte die erfolgte massenmediale Verbreitung von Bildern aus und von den Kolonien (vgl. Jäger 2010). Aus dem Blickwinkel der Kolonien aber erfuhr auch der Raum, der als Heimat angesprochen wurde, eine immense Ausdehnung. Während für viele Deutsche das Heimaterleben eine überschaubare, lokal eng umrissene räumliche Ausdehnung besaß, und die Nation lediglich als Zusammenschluss einer Vielzahl von lokalen Heimaten wahrgenommen wurde, konnte aus der Ferne die Nation selbst, das Deutsche Reich, zur Heimat werden (vgl. Petri 2001, 106).

Wenn die Verortung von, Heimat' entlang der Dimensionen von Raum, Zeit und Identität erfolgt (vgl. Gebhard/Geisler/Schröter 2007, 10), dann liegt Hellpachs Betonung, wie in den allermeisten Überlegungen zur Heimat, auf der identitätsstiftenden Funktion des Raumes. Die zeitliche Dimension wird von Hellpach $(1935,218)$ nur implizit angesprochen. Die in der Fremde als verloren empfundene Heimat ist hier wie so oft in der Kindheit angesiedelt, in der, guten alten Zeit' vor dem Eintritt ins Erwachsenenalter, in der die Umwelt noch als selbstverständlich gegeben und stabil erfahren wurde. Mit Blick auf diese gängige zeitliche Verortung ließe sich von ,Heimat' als einem generationellen Konzept sprechen, dessen zeitlicher Horizont die Lebensspanne eines Menschen umfasst und dessen Gehalt von jeder Generation neu bestimmt wird. Was bei Hellpach in der Rede vom "Heimweh nach exotischen Landschaften" aber auch anklingt, ist die zeitliche Verortung der Heimat in der nahen Zukunft. Heimat ist in dieser Konzeption nicht prekär aufgrund der Tatsache, dass sie bereits verloren ist, sondern, weil sie erst noch realisiert werden muss. Dieses Konzept einer in naher Zukunft zu realisierenden Heimat gewann mit dem Eintritt in das Zeitalter des Imperialismus zusehends an Gewicht. Christian Geulen (2003, 51), der sich mit der Frage der Dehnbarkeit der ,Heimat' im Zeitalter des Imperialismus befasste, unterschied einen älteren, im Nationalstaat gipfelnden Nationalismus vom modernen, imperialistischen Nationalismus wie folgt: "An die Stelle der Erfindung einer nationalen Vergangenheit trat die Projektion einer nationalen Zukunft, an die Stelle des (möglichen) Kriegs gegen nationale Feinde trat der ewige und alltägliche Kampf der Rassen, und an die Stelle nationaler Integration und Abgrenzung trat die koloniale Expansion und Eroberung." Der historische Imperialismus mag zwar zu seinem Ende gekommen sein, im Genre der science fiction sieht Geulen (2003, 37) die von ihm für das Zeitalter des Imperialismus ausgemachte Entgrenzung der Heimat weiter vorangetrieben. In den unendlichen Weiten des deep space "wird die Suche nach dem Eigenen in der Fremde zur eigentlichen Heimat, ist Expansion alles".

Das Heimaterleben im Deutschen Reich erfuhr, das hat die Forschung hinlänglich gezeigt, mittels der Erfahrung - und sei sie auch nur medial vermittelt - von Fremde und Exotik in den Kolonien eine nie dagewesene räumliche Expansion. Den zeitlichen Grenzen der Heimat hingegen hat die Forschung wenig Aufmerksamkeit gewidmet. Obwohl die Entdeckung und Entfaltung der geologischen Zeitskala im 19. Jahrhundert neue, unvorstellbar große Zeiträume bereitstellte, blieben der Heimat scheinbar enge zeitliche Grenzen gesetzt. Im Folgenden möchte ich mithilfe zweier Fallbeispiele - Alfred Götzes szenischem Vortrag Die Urzeit des Menschen (1898) und den populärwissenschaftlichen Schriften Die Deutsche Landschaft in Vergangenheit und Gegenwart (1915) und Das Leben der Urwelt (1931) von Wilhelm Bölsche - der Frage nachgehen, ob im Deutschen Reich parallel zur räumlichen Expansion der Heimat auch eine zeitliche Expansion erfolgte, die über die Verortung der Heimat in der nahen Vergangenheit, insbesondere der Kindheit, hinausreicht. Kann analog zur Rede von der Heimat im deep space von einer Heimat in der deep time gesprochen werden? Und falls eine solche Heimat in den unvorstellbar großen Zeiträumen der geologischen Zeitskala tatsächlich gefunden wurde, welche Funktion kam ihr zu? Wiesen prähistori- 
sche Landschaften genügend "Exotik" auf, ermöglichten sie ein Erleben von Fremdheit in einem ausreichenden Maße, um aus der zeitlichen Distanz die Gegenwart als Heimat neu bewusst erleben zu lassen? Und handelte es sich, um bei der Unterscheidung von Geulen zu bleiben, um die Erfindung einer nationalen Vergangenheit zwecks Identitätsstiftung im Rahmen des alten Nationalismus, oder wurde die Suche nach dem Eigenen in der deep time im imperialistischen Sinne zur eigentlichen Heimat?

\section{Alfred Götze. Graben nach der Urheimat}

Am Montag, den 12. Dezember 1898, abends um 8 Uhr lud das Theater der Urania in der Taubenstraße in Berlin zu einem "Scenischen Vortrag" mit dem Titel Die Urzeit des Menschen. Bilder aus den frühesten Tagen unserer Heimat. Geschrieben hat in Alfred Götze (1865-1948), ein junger Prähistoriker, der 1891 in Jena als erster in Urund Frühgeschichte promovierte, 1894 an den Grabungen in Troja teilnahm und im Anschluss eine Stelle als Assistent in der Prähistorischen Abteilung des Königlichen Museums für Völkerkunde zu Berlin annahm (vgl. Büttner 1999). Die Dioramen zu den 14 Bildern des "Scenischen Vortrags", die mittels Lichteffekten und aufwendiger Bühnentechnik die Urzeit eindrucksvoll in Szene setzten, stammten von den Malern Heinrich Harder und Wilhelm Kranz. ${ }^{1}$ Diese beiden haben mit ihren Darstellungen prähistorischer Landschaften und Lebewesen für die Urania, verschiedene populärwissenschaftliche Schriftenreihen, massenmediale Formate wie Sammelkarten sowie Auftragsarbeiten u.a. für das Deutsche Museum in München und das Aquarium Berlin die Urzeitimagination im Deutschen Reich maßgeblich geprägt. In der Druckfassung des Vortrags, die im gleichen Jahr auch im Verlag der Urania erschien, finden sich die an diesem Abend gezeigten Bilder nicht abgedruckt.

Der Vortrag nahm seine Zuhörer mit auf eine imaginäre Zeitreise, die vom ersten gesicherten Auftritt des Menschen in der Erdgeschichte bis

1 Zur technisch-medialen Beschaffenheit der ,Scenischen Vorträge' an der Urania vgl. Becker 2011, 289-292. zu jenem Zeitpunkt reichte, "wo er in unserer Heimat in das volle Licht der Geschichte eintritt" (Götze 1898, 4). Die Koordinaten dieser Zeitreise entnahm Götze (1898, 4f.) nicht der "spekulativen" Evolutionstheorie, sondern der "empirischen" Spatenforschung. Es sind folglich die im Boden verwahrten Spuren und Artefakte menschlicher Kultur, die an diesem Abend den Weg in die frühesten Tage der Heimat weisen. Ein Wissen davon, was die ,Heimat' in ihrer räumlichen Ausdehnung umfasst, wird dabei vorausgesetzt. Die Grenzen des heimatlichen Bodens müssen bereits abgesteckt sein, um im Erdreich nach seiner Tiefendimension graben zu können. Was nicht bedeutet, dass sich die Vorstellung, die sich die Zuhörer und Leser von der ,Heimat' machten, durch ihre Erkundung in der Zeit nicht ändern konnte (und sollte).

Das erste und älteste Bild aus der Heimat zeigt eine Ansiedelung bei Taubach in Thüringen im Diluvium und reicht damit verschiedenen Schätzungen zufolge zwischen 20'000 und $364^{\prime} 000$ Jahre zurück in die Vergangenheit (Götze 1898, 15). Die Technik für die genauere Bestimmung absoluter Zeitmaße sollte erst das 20. Jahrhundert liefern. Das ausgerechnet südöstlich von Weimar in Thüringen, diesem zentralen Schauplatz deutscher Geschichte und Kultur, die Anfänge menschlichen Lebens in der ,Heimat' verortet sind, verspricht eine ungebrochene Kontinuität von menschlichen Aktivitäten und ihrer geographischen Verortung von der Altsteinzeit bis ins 19. Jahrhundert. Die diluviale Landschaft aber wich vom gegenwärtigen Landschaftsbild als zentralem Träger von heimatlicher Identität deutlich ab. Wo "jetzt das Dorf Taubach liegt", beginnt der Vortrag, und spielt damit gleich zu Beginn mit der disruptiven Kraft langer Zeiträume, "zog sich damals ein langgestreckter Binnensee hin" (Götze 1898, 6). Die an seinem Ufer lagernden Menschen jagten Hirsche, Rehe und Wildschweine, aber eben auch Elefanten, Nashörner und Höhlenlöwen. In diesem und in den folgenden Bildern bricht immer wieder das Fremde und Exotische der heimatlichen Landschaft aus den frühesten Tagen in die als bekannt vorausgesetzte ,Heimat' der Zuhörer und Leser ein. Im Vortrag selbst kam durch die Wahl der Bilder und mittels ihrer Erläuterung die Heimat und damit verbunden das Heimaterleben in drei verschiedenen räumlichen Ausdehnungen und Intensitäten 
vor. 1) ,Unser Norden' als flächenmäßig kleinste, aber am intensivsten als solche erlebte ,Heimat', in der sich viele der Zuhörer in der Taubenstraße in Berlin wiederfinden durften. 2) ,Deutschland', seit 1871 Nationalstaat unter Führung ebenjenes ,Nordens', nämlich Preußens, die im europäischen Vergleich spät Realität gewordene nationale Heimat. 3) Die Vorstellung des deutschen Sprachraums als Heimat, die Bilder aus Österreich und der Schweiz mit einschließt.

Die Irritation der als bekannt vorausgesetzten ,Heimat' durch die Bilder aus den frühesten Tagen ebenjener Heimat endet erst mit dem zehnten Bild. Dieses zeigt ein nordöstlich vom Harz liegendes, also in , unserem Norden' beheimatetes Germanendorf: "Im Schatten mächtiger Eichen liegt friedlich das Dorf da und reckt seine Strohdächer empor. Man könnte glauben, ein deutsches Dörfchen aus dem 19. Jahrhundert zu erblicken [...]" (Götze 1898, 46). Hier finden sich zwei der wirkmächtigsten Heimatvorstellungen um 1900 überblendet. Einmal sehen wir eine Dorfidylle, wie sie die Heimatliteratur des späten 19. Jahrhunderts hervorbrachte, die ,Heimat' unter dem Druck von Industrialisierung und Urbanisierung bevorzugt in vorindustriellen Produktions- und Lebensweisen und damit in einer verloren gegangenen Vergangenheit verortete, die für viele Zuhörer und Leser aber zugleich noch erlebte oder zumindest vom Hörensagen bekannte Kindheit war. Diese Vergangenheit konnte aber gerade in Deutschland auch bis in ein imaginiertes "Mittelalter" zurückreichen, dessen Ständegesellschaft in Zeiten tiefgreifender gesellschaftlicher Transformationsprozesse Orientierung und Sinn zu stiften versprach (vgl. Petri 2001, 84-86). Diese Dorfidyllen haben einen lokalen Charakter und gleichen sich, so verschieden sie auch ausfallen mochten, gerade darin, dass sie als Heimat einen nur eng umrissenen, als natürlich gegeben erfahrenen Horizont einschließen.

Mit der Reichsgründung 1871 wuchs allerdings der Druck, das Gebilde auch im Inneren zu konsolidieren und der deutschen Nation eine über die lokalen und regionalen Identitäten, die vielen kleinen ,Heimaten' hinausgehende konsensfähige historische Tradition, die eine, deutsche Heimat' zu geben. Heimaterleben und Nationalismus gingen mit der Gründung des Nationalstaats eine innige Verbindung ein und waren fortan gleichermaßen Gegenstand der ,invention of tradition'. Zu diesem Zeitpunkt lässt sich ein gesteigertes
Interesse am Germanenmythos feststellen. Die Heimatbewegung des ausgehenden 19. Jahrhunderts machte in ihrer die Vergangenheit verklärenden Reaktion auf die Moderne nicht Halt beim deutschen Dörfchen in vorindustriellen Zeiten. Auf der Suche nach natürlichen Verhältnissen wandte sie sich auch dem Germanenmythos zu, wie er von Götze im Bild des Germanendorfes aufgegriffen und fortgeschrieben wurde. Das Bild zeigt das Germanendorf kurz bevor "Deutschland aus der geschichtslosen Ruhe eines prähistorischen Volkes hinaustritt auf den Schauplatz der Weltgeschichte, auf dem es unter Strömen von Blut die größten Kulturaufgaben unseres Erdteiles zu lösen berufen war [...]" (Götze 1898, 48). Es ist alles vorhanden, was den Germanenmythos im Rahmen der nationalistischen Vereinnahmung um 1900 auszeichnet. ${ }^{2}$ Germanen und Deutsche werden gleichgesetzt, es handelt sich um „unsere germanischen Vorfahren" (Götze 1898, 45). Die Germanen sind ein noch naturhaftes, d.h. jugendliches Volk, das sich durch einen „Überschuß von Lebenskraft" auszeichnet (Götze 1898, 48). Gerade weil sie noch nicht in die Geschichte eingetreten sind und damit vor allen Verwerfungen und Überformungen der deutschen Geschichte existierten, bieten sich die Germanen allen Deutschen als gemeinsame Vorfahren an. Ihre ,Urheimat' wurde in der zweiten Hälfte des 19. Jahrhunderts von vielen Anthropologen und Archäologen neu im mittleren oder nördlichen Europa, und nicht länger im Kaukasus verortet. Also ebendort, wo das Deutsche Reich Gestalt angenommen hat und eine neue Heimat bieten möchte. Außerdem treten die Germanen „das Erbe des römischen Weltreiches" (Götze 1898, 48) an und erhalten damit einen weltgeschichtlichen Auftrag, den das Deutsche Reich dankbar annimmt und weiterführt. Denn der "Kampf ums Dasein", so endet der Vortragsabend in der Taubenstraße, "zwingt auch uns heute, für die noch immer [...] überquellende und überschüssige Lebenskraft germanischen Volkstums neue Gebiete zur Bethätigung ihrer Lebensinteressen zu suchen" (Götze 1898, 62f.).

Die Irritation der als bekannt vorausgesetzten ,Heimat' durch die Bilder aus den frühesten Tagen, in denen das heimatliche Erscheinungsbild

2 Form und Funktion des Germanenmythos im Deutschen Reich sind Kipper 2002 und Wiwjorra 2006 entnommen. 
sich mehrfach radikal wandelte, endet mit dem Auftritt des germanischen Volkes auf der Bühne der Weltgeschichte. Hier hat die Landschaft eine Form angenommen, deren Kontinuität bis zur einsetzenden Industrialisierung im 19. Jahrhundert als ungebrochen vorgestellt wird, weswegen das Germanendorf einem deutschen Dörfchen aus dem 19. Jahrhundert zum Verwechseln ähnlich sieht. Die Entwicklung, die sich in den Bildern aus den frühesten Tagen vollzieht, kann als ein Erwachen der ,Heimat' zu der ihr eigenen Form beschrieben werden, die endlich in der ,Urheimat' der Germanen einen bleibenden Ausdruck findet. Die imaginäre Zeitreise in die ferne Vergangenheit hilft, die Heimat der Deutschen verstanden als nationale Einheit in der Tiefe der Zeit zu verorten und damit zu stabilisieren. In der ,Urheimat' findet die von Rolf Petri für das Deutsche Reich ausgemachte gleichermaßen inflationäre Verwendung des Wortes Heimat und des Präfixes Ur-, das von der Sehnsucht nach einem „jenseits der Zeitgrenze liegenden Ort ohne Ent-Fremdendes und Un-Eigentliches" (2001, 92) zeugt, zusammen. Die Landschaft, die bei Götze erst einmal Zeugnis von einem radikalen historischen Wandel ablegt, wird mit dem Eintreten der Germanen in die Geschichte in Natur überführt. Die ,Urheimat' wird zum „versteinerte[n] Ort" (2001, 92). Widerspruchsfrei ist diese von Götze im heimatlichen Boden geborgene deep time Heimat deswegen nicht. Sie beinhaltet sowohl Elemente des alten, im Nationalstaat gipfelnden Nationalismus, als auch des modernen, imperialistischen Nationalismus. Es wird zeitgleich eine nationale Vergangenheit erfunden und eine nationale Zukunft in Aussicht gestellt, die im alltäglichen Kampf ums Dasein nur aus kolonialer Expansion und Eroberung bestehen kann. Eine von Götze ausgemachte "geheimnisvolle Sehnsucht nach dem Süden" $(1898,61)$ treibt den Germanen/Deutschen zwar immer wieder an die Sonne, womit sich die kolonialen Bestrebungen des Deutschen Reichs bestätigt sehen durften, aber dort, wo die Germanen im Zuge der Völkerwanderung tatsächlich ihre ,Urheimat' verlassen und in Richtung Süden wandern, verlieren sie laut Götze "wie eine auf fremden Boden verpflanzte Blume ihre Kraft und gehen über kurz oder lang zu Grunde" $(1898,57)$. Die Heimat, die an diesem Abend im Theater der Urania mit großem technischen Auf- wand inszeniert und den Zuschauern vor Augen geführt wurde, zeigte sich prekär: zerrissen zwischen einem tief in der Zeit verorteten natürlichen Lebensraum, der nicht ungestraft verlassen wird, und ihrer für die Zukunft nicht nur möglichen, sondern gar geforderten Erweiterung.

\section{Wilhelm Bölsche. Rekonstruktion der deutschen Landschaftsseele}

Als 1915 Die Deutsche Landschaft in Vergangenheit und Gegenwart des Schriftstellers Wilhelm Bölsche (1861-1939) erschien, hatte die Heimat unter dem Eindruck des ersten Kriegsjahres an klaren Konturen gewonnen. "Heimat", schreibt der Herausgeber und Direktor der Urania Franz Goerke in seinem Vorwort, "das ist Dein Vaterland, das hast Du zu schützen gegen Deine Feinde und Neider ringsum". Zu diesem Zweck sollten über 130 Bilder von der "deutsche[n] Landschaft in ihrer unendlichen Vielseitigkeit" im Leser "Heimat und Heimatliebe" hervorrufen (Bölsche 1915a, 5). Ob den Soldaten im Schützengraben beim Gedanken an die Heimat tatsächlich die unendliche Vielseitigkeit des Vaterlands vor Augen gestanden hat, und nicht vielmehr das Lokalkolorit ihres Geburtsortes, darf bezweifelt werden, aber zumindest an der Heimatfront galt es, Nation und Heimat zur Deckung zu bringen und die Heimat in den Dienst des Krieg führenden Vaterlands zu stellen. Hierzu musste dieses deutsche Vaterland an konkreter, sinnlicher Gestalt gewinnen, und die Landschaft schien Goerke und Bölsche hierfür das richtige Medium zu sein. Sie richtet den Blick in die Vergangenheit und stellt die "Fragen nach dem ,Woher' allen Seins und allen Werdens" (Bölsche 1915a, 5) - und beantwortet sie zugleich. In diesen ersten Jahrzehnten des 20. Jahrhunderts vermittelt die Landschaft nicht mehr allein, wie das Stephan Günzel in seinen Überlegungen zur Landschaft als historisch wandelbarem Medium formulierte, "eine Gestalt des Nationalen, sondern ist zum eigentlichen Sitz der Volksseele geworden: die Landschaft ist Heimat" (2007, 238).

Hatte Götze noch jene Zeiträume im Blick, aus denen Spuren menschlicher Tätigkeit über- 
liefert sind, greift Bölsche auf der Suche nach der "deutsche[n] Landschaftsseele" (1915a, 21) auch auf jene Jahrmillionen der Erdgeschichte aus, die ohne den Menschen ausgekommen sind:

\begin{abstract}
Schauen wir mit dem Auge des Geologen nun in unsere deutsche Landschaft [...]. Auch diese deutsche Landschaftsseele hat in der unendlichen Dauer ihrer Vorgeschichte schon eine Art solcher "Seelenwanderung" durchgemacht, auf der sie wechselnd bald mehr dieses, bald mehr jenes Antlitz der andern irdischen Landschaften von heute trug. Tropenwald und Polaröde, Korallengrund und Wüste waren auch einmal bei uns. (1915a, 21)
\end{abstract}

Was Götze bereits in einzelnen, aber längst nicht allen Bildern seiner Urzeit des Menschen zur Anwendung brachte, nämlich den veranschaulichenden Vergleich von prähistorischen Landschaften mit ,Bildern' aus der Gegenwart, wird bei Bölsche zum Programm. Jede prähistorische Deutsche Landschaft hat in der Gegenwart ihre Entsprechung. Dergestalt können Zeit und Raum aufeinander abgebildet werden; die imaginäre Zeitreise in die Vergangenheit der Deutschen Landschaft entpuppt sich als ein Reise um den Globus. Das Heimweh nach dem Süden, nach Sonne und Exotik, das neben Hellpach und Götze auch Bölsche den Deutschen attestiert, findet damit ein neues Objekt der Sehnsucht: es liegt nicht mehr länger "[i]rgendwo und nirgendwo fern im Süden", sondern ist "ein Urbild unserer deutschen Heimat" (1915a, 22). Anstelle einer Reise nach Kaiser-Wilhelms-Land tut es auch ein Blick in die Deutsche Landschaft zur Jurazeit, von der die "australische Korallensee im Umkreise des australischen Festlandes, Neu-Guineas und der benachbarten weit in die blaue Ozeanfläche ausschwärmenden Korallenarchipele" ein anschauliches Bild gibt (Bölsche 1915a, 49). Dergestalt gibt die ferne Vergangenheit der Heimat Aufschluss darüber, ob die im Rahmen der deutschen Expansion annektierten Gebiete zur zukünftigen Heimat werden können. Heimat, geben die prähistorischen Landschaften des Deutschen Reichs all jenen zu verstehen, die ihre "sichtbaren Spuren noch in dem Heutigen" (Bölsche 1915a, 71) zu lesen verstehen, kann soviel mehr sein, und in einem expandierenden Kolonialreich wird die Suche nach dem Eigenen in der Fremde zur eigentlichen Heimat. Aus diesem Grund dürfte Goerke die „Frage nach den Schicksalen der Erde" in seinem Vorwort einigermaßen überraschend $\mathrm{zu}$ den wichtigsten Fragen überhaupt gezählt haben, die auch in Kriegszeiten "einen jeden denkenden Menschen beschäftigen müssen" (Bölsche 1915a, 5).

Voraussetzung dieser imperialistischen Lektüre prähistorischer Landschaften ist die Annahme, dass Raum und Zeit ein Kontinuum bilden, in dem die Rede von ,Deutschland' Sinn macht. Wie abenteuerlich diese Annahme ist, zeigt die „Erd-Karte der älteren Urwelt", die dem Text als Initiale beigegeben ist (Abb. 1).

Die Karte zeigt den nördlichen und den südlichen Superkontinent zur Zeit des Devon, durch die Tethys voneinander getrennt. Dieser Urweltkarte ist eine zweite, aktuelle Weltkarte hinterlegt. Mittels dieses Konstrukts kann Bölsche in der Devonzeit das Meer „über Deutschland

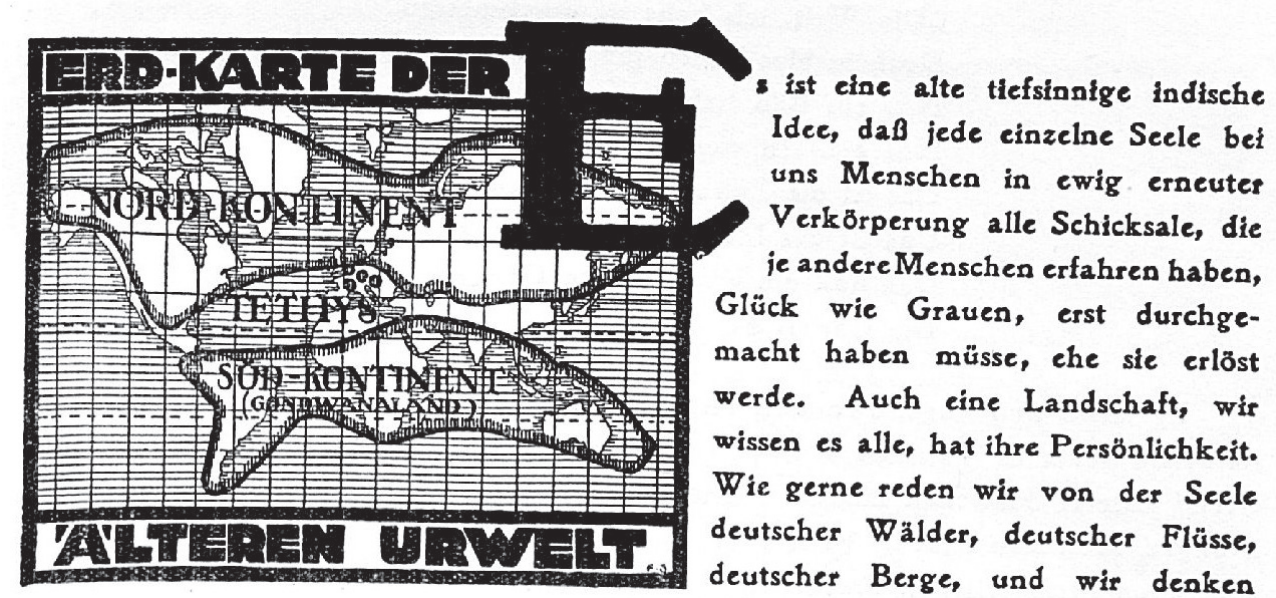

Abb. 1: Erd-Karte der älteren Urwelt (aus Bölsche 1915a, 21). 
blauen" (1915a, 21) lassen. Nur: so richtig es ist, dass sich überall auf dem Gebiet des Deutschen Reichs marine Sedimente finden, so fiktiv ist das Kontinuum, das diese Kartenkonstruktion zu evozieren sucht. Was für die von Götze in den Blick genommenen Ur- und Frühgeschichtlichen Zeiträume weitgehend gilt, die territoriale Kontinuität ihrer Spuren und Artefakte, gilt nicht gleichermaßen für die Zeiträume der Erdgeschichte. Was 1915 im Deutschen Reich an Sedimenten zu finden ist, konnte zur Zeit des Devon ganz woanders abgelagert worden sein. Die Rede von den prähistorischen Landschaften des Deutschen Reichs ist folglich eine gewaltige Konstruktionsleistung. Die Suggestion von territorialer Integrität über alle Revolutionen der Erdgeschichte hinweg trägt erheblich zur Naturalisierung und Stabilisierung politischer Ansprüche und Grenzen bei.

Wie eine solche prähistorische Landschaft ausgesehen haben mag, zeigt das Frontispiz zu Bölsches Das Leben der Urwelt (1931), das die Leser "in fremde Zonen und Meere, zu geheimnisvollen Wäldern und seltsamster Tierwelt [führt], kaum daß wir unsere engste Heimat dafür zu verlassen brauchen" (3). Es gehört zu den zahlreichen Werken, mit denen Bölsche wie kein anderer die Evolutionstheorie von Charles Darwin und deren Adaption durch Ernst Haeckel im Deutschen Reich als Weltdeutungsentwurf populär gemacht hat. Und zwar nicht in Form eines kalten Sozialdarwinismus, sondern in einer naturphilosophischidealistischen Form, die den ,Geist' bereits in der Natur am Werk sieht, bis er endlich im Menschen zu sich selbst kommt. Philipp Sarasin und Michael Hagner haben Bölsches Weltsicht zutreffend als "Darwin mit Goethe, Schelling und Hegel" (2008, $57)$ beschrieben. Weil Evolution in Bölsches Auslegung dadurch "nichts anderes als die Bildungsgeschichte des Menschen" ist, kann er in Das Leben der Urwelt schreiben: "Auch in diesen Sauriern hat sich ein weltgeschichtlicher und geistesgeschichtlicher Gedanke der Natur einmal ausgelebt, wie er in uns schafft und wirkt." (Bölsche 1931, 298)

In diesem populärwissenschaftlichen Werk rekonstruiert Bölsche die deep time Heimat, wo er zuvor lediglich ihren Spuren nachging. Wo Die Deutsche Landschaft in Vergangenheit und Gegenwart den Hohenzollern zeigt (Abb. 2), einen Zeugenberg der zum Juragebirge gehörenden Schwäbischen Alb, auf dessen Gipfel die Stamm- burg des Preußischen Kaiserhauses thront, zeigt das Frontispiz zu Das Leben der Urwelt (Abb. 3) an ebenjener Stelle eine exotische Südseelandschaft.

Im Vordergrund des von Hugo Wolff-Maage gefertigten Bildes, das die Juraperiode zeigt, auf deren Sedimenten dereinst die Burg Hohenzollern thronen wird, jagen zwei Plesiosaurier, ein erwachsenes Tier mit Nachwuchs, nach Nahrung. In nuce bringt das Frontispiz zur Anschauung, wofür das ,Leben der Urwelt' bei Bölsche Modell steht: für Reproduktion und Nahrungsaufnahme, für einen voll entfesselten, noch nicht sozial eingehegten Kampf ums Dasein, in welchem dennoch bereits der ,Geist' sichtbar ist. Im Hintergrund rechts ist der Antagonist des Plesiosaurus zu sehen, ein Ichthyosaurier. Die bei Holzmaden auf der Schwäbischen Alb, nicht weit vom Hohenzollern zuhauf gefundenen fossilen Überreste des Ichthyosaurus machten diesen zu einem echt deutschen Saurier, den Bölsche "natürlichen U-Boot[en]" gleich "auf die ozeanische Erdumsegelung [sich] begeben" lässt, um "wie tapfere Ritter gegen fremdes Sauriervolk" zu ziehen, wobei sie von "Schwaben bis Indien und Australien gekommen" (1931, 94) sind. Diese Analogie erschöpft sich bei ihm nicht im Metaphorischen. Aufgrund seiner speziellen Auslegung der Evolutionstheorie sieht er die Natur im Ichthyosaurus tatsächlich auf niedriger Stufe erreichen, was deutsche Ingenieurskunst mit dem U-Boot auf ungemein höherer Stufe wieder schaffen sollte. Weil in Bölsches Weltdeutung wie er bereits in Der Mensch der Zukunft schrieb - jedes Tier "bis zum Ichthyosaurus hinab" (Bölsche 1915b, 63) noch einmal Mensch wird, insofern dieser sich seinen Entwicklungsgang vergegenwärtigt, kann der Ichthyosaurus hier tatsächlich zum Vorfahren des Deutschen werden, hat das Deutsche Reich von 1931 mitsamt seiner Marine im "Saurierland" (Bölsche 1931, 15) von vor Jahrmillionen seine Entsprechung. Dergestalt transformiert die Urwelt bei Bölsche zu einem ins Symbolische verschobenen Schauplatz imperialistischer Phantasmen, der Leser braucht die "engste Heimat" nicht zu verlassen, um „in fremde Zonen und Meere" (Bölsche 1931, 15) vorzudringen. Diese ,Heimat' ist Schauplatz der immer gleichen Kämpfe, ob in den Tagen der großen Saurier, der Germanen oder des Deutschen Reichs. 


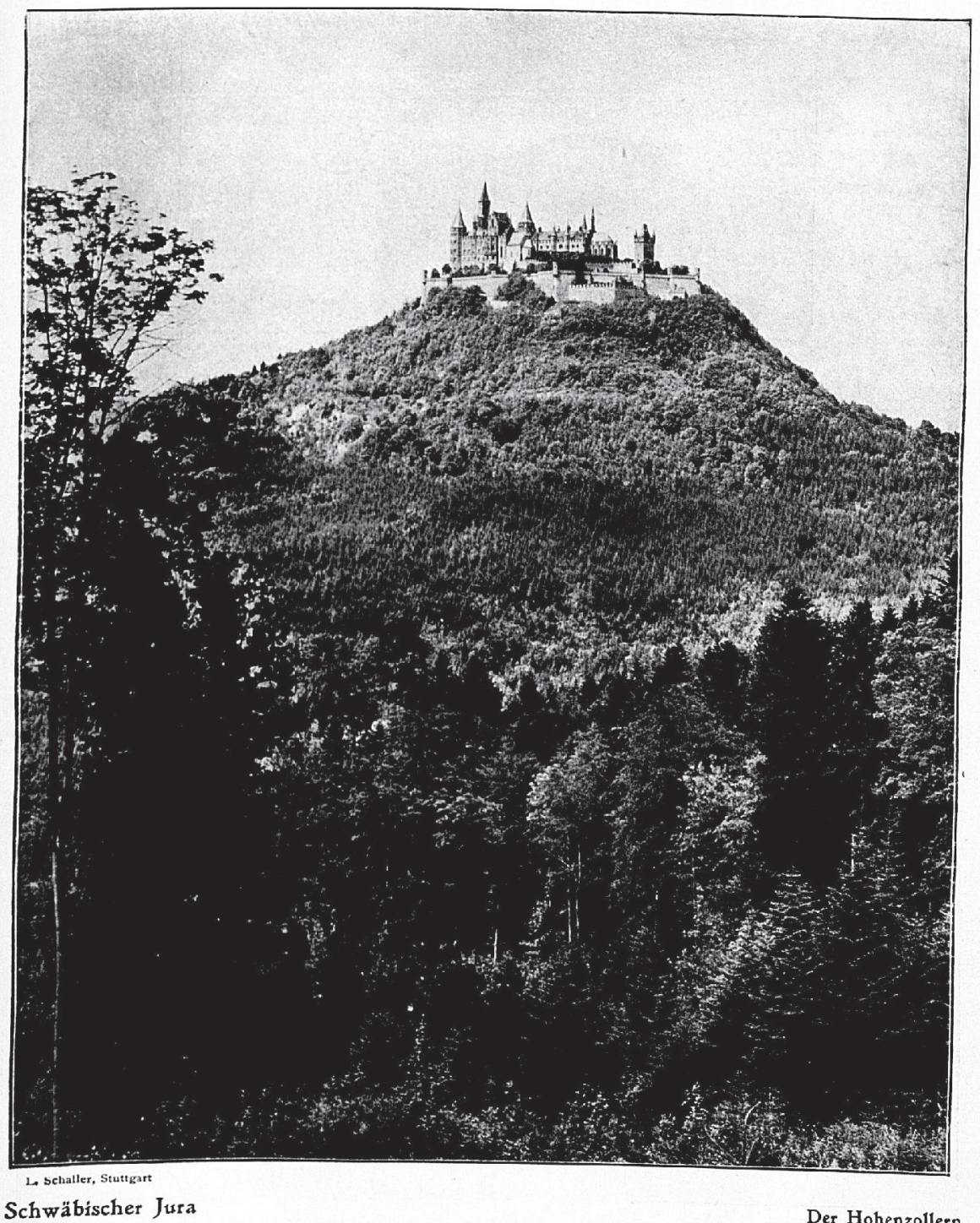

Abb. 2: Der Hohenzollern im Schwäbischen Jura (aus Bölsche 1915a, 81).

\section{Fazit. Form und Funktion der deep time Heimat}

Das Deutsche Reich, das haben die Fallbeispiele gezeigt, sah parallel zur räumlichen auch eine zeitliche Expansion der Heimat, welche über die nahe Vergangenheit weit hinausreichte und über die Ur- und Frühgeschichte bis tief in die Erdgeschichte ausgriff. Die Notwendigkeit dieser zeitlichen Ausdehnung ergab sich einerseits aus der Reichsgründung 1871, infolge derer der deutschen Nation eine konsensfähige historische Tradition, eine, deutsche Heimat' zuwachsen musste. Das hieraus resultierende Heimaterleben im Deutschen Reich war in zuvor ungekanntem Ausmaß an einen erstarkten Nationalismus geknüpft. Heimat wurde zwar weiterhin in der Kindheit und Jugend verortet, aber an die Seite der individuellen Erinnerung trat das kollektive Gedächtnis an die Kindheit und das Jugendalter des Volkes und der Nation. Heimaterleben konnte fortan auch die ,Urheimat' der Germanen mit einschließen. Andererseits war die zeitliche Ausdehnung der Heimat eine Reaktion auf die "Tiefendimension des Lebens" (Kugler 2013, 397), die Darwin mit der Formulierung seiner Evolutionstheorie einführte. In dem Moment, wo diese zu einem umfassenden Weltdeutungsentwurf ausgebaut wird, wie das bei Bölsche der Fall war, der die Gegenwart als eine Aktualisierung des längst Vergangenen beschrieb, galt es das ,Leben' in seiner aktuellen Verfasstheit auf seinen erdgeschichtlichen Sinn hin zu befragen. 


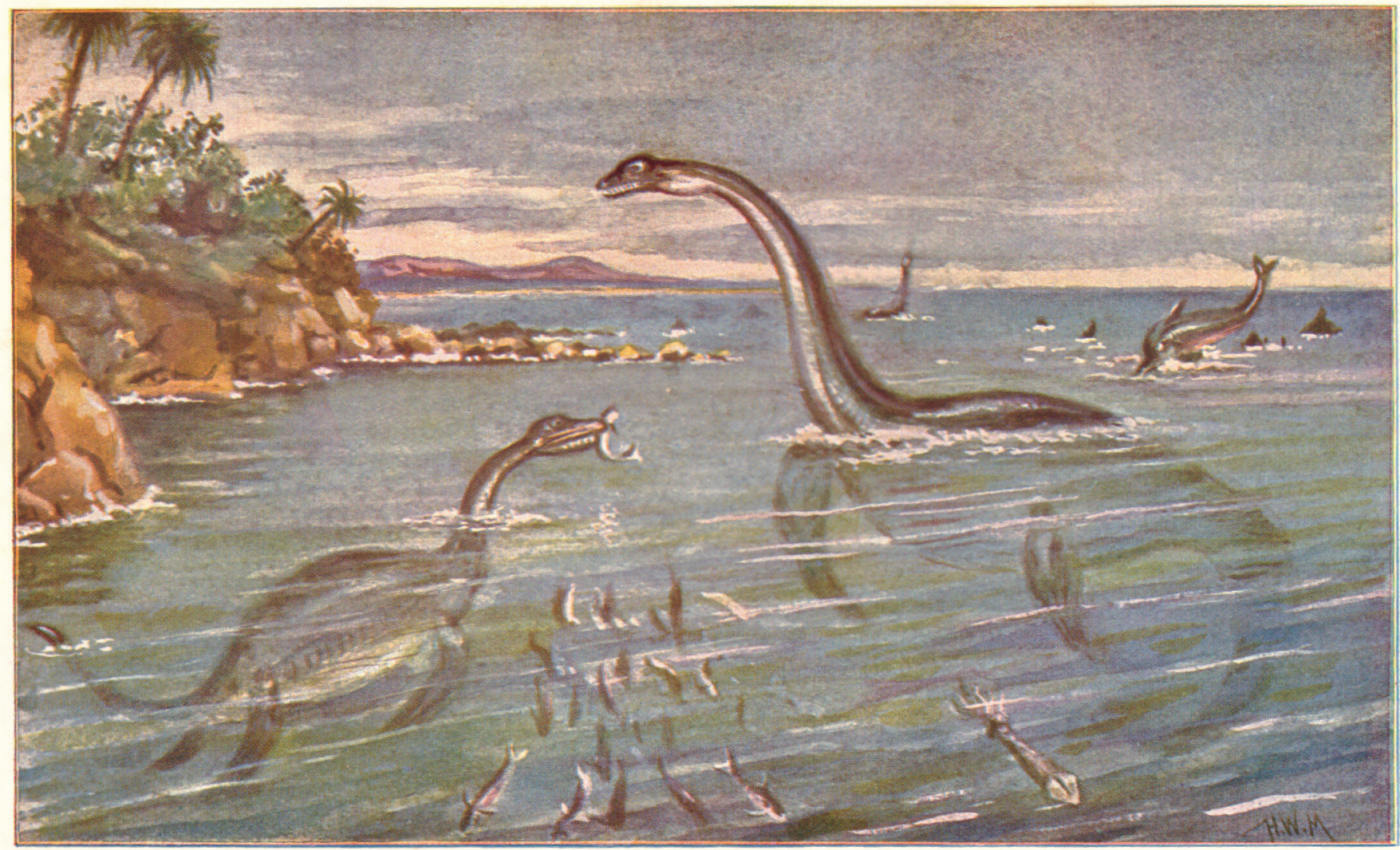

Abb. 3: Im süddeutschen Meer der Juraperiode (aus Bölsche 1931: Frontispiz).

Heimat, das zeigt Bölsches Die Deutsche Landschaft in Vergangenheit und Gegenwart ganz klar, erschloss sich den Zeitgenossen bevorzugt im Medium der Landschaft. Die im Verlauf der zeitlichen Expansion erschlossenen prähistorischen Landschaften des Deutschen Reichs waren ähnlich exotisch wie jene fremden Landschaften, die im Zuge der räumlichen Expansion das deutsche Kolonialreich zu bieten hatte. Ja mehr noch: Die Reise in die Vergangenheit der Heimat war in allen Fällen zeitgleich eine Reise um den Globus. Das Heimweh nach dem Süden, nach Sonne und Exotik, das alle behandelten Autoren den Deutschen attestierten, konnte somit gleichermaßen mit Bildern aus den Kolonien und aus der Vergangenheit der Heimat gestillt werden.

Es gab sie folglich, die Heimat in der deep time. Sie trat in zwei Versionen mit je unterschiedlichen Funktionen auf den Plan. 1) Die nationalistische deep time Heimat. In ihr enden die transformativen Kräfte der deep time zu einem Zeitpunkt, in dem Heimat ,zu sich selbst gekommen' ist. Diese fortan der Zeit enthobene Urheimat hilft, die nationale Einheit in der Tiefe der Zeit zu verorten und damit zu stabilisieren. Diese deep time Heimat kündet von den tief im Boden wurzelnden natürlichen Ursprüngen der deutschen Nation. Autoren wie Götze und Bölsche, die ihre Leser dazu anleiteten, die Spuren der Zeit in der heimischen Landschaft zu sehen und zu lesen, beförderten folglich ein umfassenderes und zugleich abstrakteres, ein ,nationales' Heimaterleben. 2) Die imperialistische deep time Heimat. Diese deep time Heimat wird, gerade weil sie lange Zeiträume in den Blick zu nehmen vermag, zum Anschauungsfall für den von beiden Autoren ausgemachten ,Kampf ums Dasein'. Expansion ist hierin die einzig mögliche Form der Weiterentwicklung. Die, Heimat' ist also immer schon auf ihre Erweiterung hin angelegt, sie muss expandieren. Was ,Heimat' ist, wird folglich erst die Zukunft zeigen.

Wirklich miteinander vereinbar sind die zwei Konzeptionen von deep time Heimat im Deutschen Reich nicht. Und doch scheinen sie komplementäre Aufgaben wahrzunehmen, sodass weder Götze noch Bölsche auf die eine oder die andere Konzeption verzichten wollen. Damit die prähistorischen Landschaften des Deutschen Reichs zum Medium der im Boden wurzelnden, gleichsam versteinerten deep time Heimat avancieren kön- 
nen, müssen die transformativen Kräfte der Erdgeschichte im Zeitraffer vor Augen geführt werden. Dadurch wiederum gerät der feste Boden, auf dem die Heimat steht, in Bewegung, sodass sie sich nur mittels Expansion erhalten lässt. Die zeitliche Expansion der Heimat im Deutschen Reich führt also dazu, das sich das Heimaterleben in einer liminalen Zeitzone abspielt, gelegen zwischen dem, was die Heimat in der Vergangenheit geworden war, und dem, was sie zu ihrer Bewahrung in Zukunft werden muss. Hierin erweist sich die deep time Heimat als prekär.

\section{Literaturverzeichnis}

\section{Quellen}

Bölsche, Wilhelm (1915a): Die Deutsche Landschaft in Vergangenheit und Gegenwart. Mit über 130 Bildern. In: Goerke, Franz (Hg.). Berlin-Charlottenburg: Vita Deutsches Verlagshaus. (= Leuchtende Stunden; 8).

Bölsche, Wilhelm (1915b): Der Mensch der Zukunft. Stuttgart: Kosmos, Gesellschaft der Naturfreunde.

Bölsche, Wilhelm (1931): Das Leben der Urwelt. Aus den Tagen der großen Saurier. Leipzig: Georg Dollheimer.

Götze, Alfred (1898): Die Urzeit des Menschen. Bilder aus den frühesten Tagen unserer Heimat. Scenischer Vortrag. Berlin: Verlag der Gesellschaft „Urania”.

Hellpach, Willy (1935): Geopsyche. Die Menschenseele unterm Einfluß von Wetter und Klima, Boden und Landschaft. Vierte, völlig neu bearbeitete Auflage der "Geopsychischen Erscheinungen". Leipzig: Wilhelm Engelmann.

\section{Forschungsliteratur}

Becker, Kristin (2011): "Welt von Wundern". Die Berliner Urania um 1900. In: Becker, Tobias/Littmann, Anna/ Niedbalski, Johanna (Hgg.): Die tausend Freuden der Metropole. Bielefeld: transcript, S. 283-302.

Büttner, Willfried (1999): Alfred Götze - Pionier der Spatenforschung und der archäologischen Denkmalpflege. In: Alt-Thüringen. Jahresschrift des Thüringischen Landesamtes für archäologische Denkmalpflege 33, S. 10-29.

Gebhard, Gunther/Geisler, Oliver/Schröter, Steffen (2007): Heimatdenken: Konjunkturen und Konturen. Statt einer Einleitung. In: Dies. (Hgg.): Heimat.
Konturen und Konjunkturen eines umstrittenen Konzepts. Bielefeld: transcript, S. 9-56.

Geulen, Christian (2003): „The Final Frontier..." Heimat, Nation und Kolonie um 1900: Carl Peters. In: Kundrus, Birthe (Hg.): Phantasiereiche. Zur Kulturgeschichte des deutschen Kolonialismus. Frankfurt/New York: Campus, S. 35-55.

Günzel, Stephan (2007): Landschaft als Medium: Historische Formen. In: Archiv für Mediengeschichte 7: Stadt - Land - Fluss. Medienlandschaften, S. 231-238.

Güttler, Nils (2017): Heimat und Ökologie: Umgebungswissen in der Botanik um 1900. In: Huber, Florian/Wessely, Christina (Hgg.): Milieu. Umgebungen des Lebendigen in der Moderne. Paderborn: Wilhelm Fink, S. 105-120.

Jäger, Jens (2009): Colony as Heimat? The Formation of Colonial Identity in Germany around 1900. In: German History 27/4, S. 467-489.

Jäger, Jens (2010): Plätze an der Sonne? Europäische Visualisierungen kolonialer Realitäten um 1900. In: Kraft, Claudia/Lüdtke, Alf/Martschukat, Jürgen (Hgg.): Kolonialgeschichten. Regionale Perspektiven auf ein globales Phänomen. Frankfurt/New York: Campus, S. 162-184.

Kipper, Rainer (2002): Der Germanenmythos im Deutschen Kaiserreich. Formen und Funktionen historischer Selbstthematisierung. Göttingen: Vandenhoeck \& Ruprecht.

Kugler, Lena (2013): Die Tiefenzeit von Dingen und Menschen. (Falsche) Fossilien und die „Bergwerke zu Falun". In: Weimarer Beiträge 59/3, S. 397-415.

Lekan, Thomas M. (2004): Imagining the Nation in Nature. Landscape Preservation and German Identity, 1885-1945. Cambridge, MA/London: Harvard University Press.

Miller, Rudolf (2018): Von Willy Hellpachs „Geopsyche" bis heute. Eine Spurensuche in der Psychologie. In: Braungart, Georg/Büttner, Urs (Hgg.): Wind und Wetter. Kultur - Wissen - Ästhetik. Paderborn: Wilhelm Fink, S. 333-356.

Petri, Rolf (2001): Deutsche Heimat 1850-1950. In: Comparativ 11/1, S. 77-127.

Sarasin, Philipp/Hagner, Michael (2008): Wilhelm Bölsche und der „Geist". Populärer Darwinismus in Deutschland 1887-1934. In: Nach Feierabend. Zürcher Jahrbuch für Wissensgeschichte 4. Darwin. Zürich/Berlin: diaphanes, S. 47-67.

Wiwjorra, Ingo (2006): Der Germanenmythos. Konstruktion einer Weltanschauung in der Altertumsforschung des 19. Jahrhunderts. Darmstadt: Wissenschaftliche Buchgesellschaft. 